\title{
Tests for Skewness, Kurtosis, and Normality for Time Series Data
}

\author{
Jushan Bai * Serena $\mathrm{Ng}^{\dagger}$
}

June 2001

\begin{abstract}
We present the sampling distributions for the coefficient of skewness, kurtosis, and a joint test of normality for time series observations. In contrast to independent and identically distributed data, the limiting distributions of the statistics are shown to depend on the long run rather than the short-run variance of relevant sample moments. Monte Carlo simulations show that the test statistics for symmetry and normality have good finite sample size and power. However, size distortions render testing for kurtosis almost meaningless except for distributions with thin tails such as the normal distribution. Nevertheless, this general weakness of testing for kurtosis is of little consequence for testing normality. Combining skewness and kurtosis as in Bera and Jarque (1981) is still a useful test of normality provided the limiting variance accounts for the serial correlation in the data.
\end{abstract}

JEL Classification: C12, C22

Keywords: symmetry, kurtosis, normality, Bera-Jarque test.

\footnotetext{
${ }^{*}$ Department of Economics, Boston College, Email: jushan.bai@bc.edu.

${ }^{\dagger}$ Department of Economics, Boston College. Email: serena.ng@bc.edu Correspondence: Serena Ng, Department of Economics, Boston College, Chestnut Hill, MA, 02467.
} 


\section{Introduction}

Consider a series $\left\{X_{t}\right\}_{t=1}^{T}$ with mean $\mu$ and standard deviation $\sigma$. Let $\mu_{r}=E\left[(x-\mu)^{r}\right]$ be the $r^{t h}$ central moment of $X_{t}$ with $\mu_{2}=\sigma^{2}$. The coefficient of skewness and kurtosis are defined as:

$$
\begin{aligned}
\tau & =\frac{\mu_{3}}{\sigma^{3}}=\frac{E\left[(x-\mu)^{3}\right]}{E\left[(x-\mu)^{2}\right]^{3 / 2}} \\
\kappa & =\frac{\mu_{4}}{\sigma^{4}}=\frac{E\left[(x-\mu)^{4}\right]}{E\left[(x-\mu)^{2}\right]^{2}} .
\end{aligned}
$$

Sample estimates of $\tau$ and $\kappa$ can be obtained upon replacing population moments $\mu_{r}$ by the sample moments $\widehat{\mu}_{r}=T^{-1} \sum_{t=1}^{T}\left(X_{t}-\bar{X}\right)^{r}$. If $X_{t}$ is $i . i . d$. and normally distributed, then $\sqrt{T} \widehat{\tau} \stackrel{d}{\longrightarrow} N(0,6)$ and $\sqrt{T}(\widehat{\kappa}-3) \stackrel{d}{\longrightarrow} N(0,24)$, [see, e.g. Kendall and Stuart (1969)]. This paper presents the limiting distributions for $\widehat{\tau}$ and $\widehat{\kappa}$ when the data are weakly dependent.

Whether time series data exhibit skewed behavior has been an issue of macroeconomic interest. Some authors [e.g. Neftci (1994) and Hamilton (1989)] use parametric models to see if economic variables behave similarly during expansions and recessions. Others use simple statistics to test skewness. In a well-known article, Delong and Summers (1985) studied whether business cycles are symmetrical by applying the skewness coefficient to GDP, industrial production, and the unemployment rate. However, because the sampling distribution of the skewness coefficient for serially correlated data is not known, the authors obtained critical values by simulating an AR(3) model with normal errors. These critical values are correct only if the $\operatorname{AR}(3)$ model is the correct data generating process and that the errors are indeed normal. The results developed in this article allows us to test for symmetry without making such assumptions.

The coefficient of kurtosis is informative about the tail behavior of a series, an issue which has drawn substantial interest in the finance literature. However, we argue that measuring tails behavior by using kurtosis is not a sound approach. As will be shown below, the true value of $\kappa$ is, in general, substantially underestimated. A very large number of observations is required to get a reasonable estimate. This bias translates into size distortion for testing kurtosis. Exceptions are distributions with thin tails, such as the normal distribution. But in such cases, concerns for heavy tails are quite rare. As such, testing for kurtosis is not a very useful exercise per se.

If $X_{t}$ is symmetrically distributed, $\mu_{3}$ and thus $\tau$ will be zero. The Gaussian distribution has $\tau=0$ and $\kappa=3$. When $\kappa>3$, the distribution of $X_{t}$ is said to have fat tails. Normality is often a maintained assumption in estimation and finite sample inference. A joint test of $\tau=0$ and $\kappa-3=0$ is often used as a test of normality. Bera and Jarque $(1981)$ showed that $T\left(\widehat{\tau}^{2} / 6+(\widehat{\kappa}-3)^{2} / 24\right) \stackrel{d}{\longrightarrow}$ $\chi_{2}^{2}$. We extend their results developed for i.i.d. data to weakly dependent data. 


\section{The Test Statistics}

For any integer $r \geq 1$, we first note the following:

$$
\begin{aligned}
\frac{\widehat{\mu}_{r}}{\widehat{\sigma}^{r}}-\frac{\mu_{r}}{\sigma^{r}} & =\frac{\left(\widehat{\mu}_{r}-\mu_{r}\right)}{\widehat{\sigma}^{r}}-\frac{\mu_{r}}{\sigma^{r}}\left[\frac{\left(\widehat{\sigma}^{2}\right)^{r / 2}-\left(\sigma^{2}\right)^{r / 2}}{\widehat{\sigma}^{r}}\right] \\
& =\left[\frac{T^{-1} \sum_{t=1}^{T}\left(X_{t}-\bar{X}\right)^{r}-\mu_{r}}{\widehat{\sigma}^{r}}\right]-\frac{\mu_{r}}{\sigma^{r}}\left[\frac{\left(\left(\widehat{\sigma}^{2}\right)^{r / 2}\right)-\left(\left(\sigma^{2}\right)^{r / 2}\right)}{\widehat{\sigma}^{r}}\right] .
\end{aligned}
$$

Lemma 1 in the Appendix provides large sample approximations to the normalized central moment. This is used to obtain the sampling distributions of $\widehat{\tau}$ and $\widehat{\kappa}$.

\subsection{Testing for Skewness}

We first derive the limiting distribution of the estimated skewness coefficient under arbitrary $\tau$ (not necessarily zero) and then specialize the general result to $\tau=0$. Throughout, we assume that the central limit theorem holds for the $4 \times 1$ vector series $W_{t}^{\prime}=\left[X_{t}-\mu,\left(X_{t}-\mu\right)^{2}-\sigma^{2},\left(X_{t}-\mu\right)^{3}-\right.$ $\left.\mu_{3},\left(X_{t}-\mu\right)^{4}-\mu_{4}\right](t=1, \ldots, T)$. This requires finite $(8+\delta)$ th $(\delta>0)$ moment and some mixing conditions. When testing symmetry, finite $(6+\delta)$ th moment and some mixing conditions will be sufficient.

Theorem 1 Suppose $X_{t}$ is stationary up to sixth order. Then

$$
\begin{aligned}
\sqrt{T}(\widehat{\tau}-\tau) & =\frac{\alpha}{\widehat{\sigma}^{3}} \frac{1}{\sqrt{T}} \sum_{t=1}^{T} Z_{t}+o_{p}(1), \\
\text { where } \alpha & =\left[1-3 \sigma^{2}-\frac{3 \sigma \tau}{2}\right], \quad Z_{t}=\left[\begin{array}{c}
\left(X_{t}-\mu\right)^{3}-\mu_{3} \\
\left(X_{t}-\mu\right) \\
\left(X_{t}-\mu\right)^{2}-\sigma^{2}
\end{array}\right],
\end{aligned}
$$

and $\frac{1}{\sqrt{T}} \sum_{t=1}^{T} Z_{t} \stackrel{d}{\longrightarrow} N(0, \Gamma)$, where $\Gamma=\lim _{T \rightarrow \infty} T E\left(\bar{Z} \bar{Z}^{\prime}\right)$ with $\bar{Z}$ being the sample mean of $Z_{t}$. Moreover,

$$
\sqrt{T}(\widehat{\tau}-\tau) \stackrel{d}{\longrightarrow} N\left(0, \frac{\alpha \Gamma \alpha^{\prime}}{\sigma^{6}}\right) .
$$

Serial dependence in $X_{t}$ is explicitly taken into account through $\Gamma$, the spectral density matrix at frequency zero of $Z_{t}$. This result permits testing the skewness coefficient at any arbitrary value of $\tau$ and even when the data are serially correlated.

In the special case when $\tau=0$ (or equivalently, $\mu_{3}=0$ ), the last element of $\alpha$ is zero. Thus, one only needs to consider the sampling properties of

$$
\frac{1}{\sqrt{T}} \sum_{t=1}^{T} Z_{t}=\frac{1}{\sqrt{T}} \sum_{t=1}^{T}\left[\begin{array}{c}
\left(X_{t}-\mu\right)^{3} \\
\left(X_{t}-\mu\right)
\end{array}\right]
$$


This leads to the following result.

Corollary 1 Under the null hypothesis that $\tau=0$,

$$
\sqrt{T} \widehat{\tau} \stackrel{d}{\longrightarrow} N\left(0, \frac{\alpha_{2} \Gamma_{22} \alpha_{2}^{\prime}}{\sigma^{6}}\right)
$$

where $\alpha_{2}=\left[1,-3 \sigma^{2}\right]$ and $\Gamma_{22}$ is the first $2 \times 2$ block matrix of $\Gamma$.

Similarly, one can easily show that, under $\tau=\mu_{3}=0$,

$$
\sqrt{T} \widehat{\mu}_{3} \stackrel{d}{\longrightarrow} N\left(0, \alpha_{2} \Gamma_{22} \alpha_{2}^{\prime}\right) .
$$

The only difference between the limiting distributions of $\widehat{\mu}_{3}$ and $\widehat{\tau}$ is the normalizing constant $\sigma^{6}$. If the asymptotic standard deviations are estimated by $s\left(\widehat{\mu}_{3}\right)=\left(\widehat{\alpha}_{2} \widehat{\Gamma}_{22} \widehat{\alpha}_{2}^{\prime}\right)^{1 / 2}$ and $s(\widehat{\tau})=$ $\left(\widehat{\alpha}_{2} \widehat{\Gamma}_{22} \widehat{\alpha}_{2}^{\prime} / \widehat{\sigma}^{6}\right)^{1 / 2}$, then we have the numerical identity, $\widehat{\mu}_{3} / s\left(\widehat{\mu}_{3}\right)=\widehat{\tau} / s(\widehat{\tau})$. We summarize the above results in the following theorem.

Theorem 2 Suppose $X_{t}$ is stationary up to sixth order and let $\widehat{\alpha}_{2}=\left[1,-3 \widehat{\sigma}^{2}\right]$. Let $\widehat{\sigma}^{2}$ and $\widehat{\Gamma}_{22}$ be consistent estimates of $\sigma^{2}$ and $\Gamma_{22}$, respectively. Let $s\left(\widehat{\mu}_{3}\right)=\left(\widehat{\alpha}_{2} \widehat{\Gamma}_{22} \widehat{\alpha}_{2}^{\prime}\right)^{1 / 2}$ and $s(\widehat{\tau})=$ $\left(\widehat{\alpha}_{2} \widehat{\Gamma}_{22} \widehat{\alpha}_{2}^{\prime} / \widehat{\sigma}^{6}\right)^{1 / 2}$. Then, under the null hypothesis of $\tau=\mu_{3}=0$, we have

$$
\pi_{3}=\frac{\sqrt{T} \widehat{\mu}_{3}}{s\left(\widehat{\mu}_{3}\right)}=\frac{\sqrt{T} \widehat{\tau}}{s(\widehat{\tau})} \stackrel{d}{\longrightarrow} N(0,1) .
$$

That is, $\widehat{\mu}_{3}$ and $\widehat{\tau}$ are the same. To construct $\widehat{\pi}_{3}$, one needs only to obtain a consistent estimate of this long-run variance, such as non-parametrically by kernel estimation. The test is valid even if the null distribution is not normally distributed, albeit symmetric. Simple calculations show that if $X_{t}$ is iid normal, the variance of $\widehat{\tau}$ is 6 .

Depending on the distribution under investigation, a large number of observations might be required to detect symmetry. The possibility of low power can be remedied in two ways. The first is to exploit the fact that most economic time series are bounded below by zero. Hence, one can test symmetry against positive skewness. Second, the odd moments of symmetric distributions are zero, if they exist. Therefore, one can construct a joint test of several odd moments to increase power. To illustrate, consider a joint test of two odd moments, $r_{1}$ and $r_{2}$. Let

$$
Y_{T}=\left(\begin{array}{c}
\frac{1}{\sqrt{T}} \sum_{t=1}^{T}\left(X_{t}-\bar{X}\right)^{r_{1}} \\
\frac{1}{\sqrt{T}} \sum_{t=1}^{T}\left(X_{t}-\bar{X}\right)^{r_{2}}
\end{array}\right)
$$

By Lemma 1 in the appendix, we can show

$$
Y_{T}=\alpha \frac{1}{\sqrt{T}} \sum_{t=1}^{T} Z_{t}+o_{p}(1)
$$


where

$$
\alpha=\left[\begin{array}{ccc}
1 & 0 & -r_{1} \mu_{r_{1}-1} \\
0 & 1 & -r_{2} \mu_{r_{2}-1}
\end{array}\right], \quad Z_{t}=\left(\begin{array}{c}
\left(X_{t}-\mu\right)^{r_{1}} \\
\left(X_{t}-\mu\right)^{r_{2}} \\
\left(X_{t}-\mu\right)
\end{array}\right) .
$$

Assuming that a central limit theorem holds for $Z_{t}$ such that $\frac{1}{\sqrt{T}} \sum_{t=1}^{T} Z_{t} \stackrel{d}{\longrightarrow} N(0, \Gamma)$, where $\Gamma=\lim _{T \rightarrow \infty} T E\left(\bar{Z} \bar{Z}^{\prime}\right)$, we have $Y_{T} \stackrel{d}{\longrightarrow} N\left(0, \alpha \Gamma \alpha^{\prime}\right)$ under the null hypothesis of symmetry. Let $\widehat{\alpha \Gamma \alpha^{\prime}}$ be a consistent estimate for $\alpha \Gamma \alpha^{\prime}$ (which is easy to obtain), we then have

$$
\widehat{\mu}_{r_{1}, r_{2}}=Y_{T}^{\prime}\left(\widehat{\alpha \Gamma \alpha^{\prime}}\right)^{-1} Y_{T} \stackrel{d}{\longrightarrow} \chi_{2}^{2} \text {. }
$$

Generally, this is a more powerful test than the test based on the third moment alone. The cost is that this test requires the finiteness of $\left(2 r_{2}\right)$ th moment $\left(r_{1}<r_{2}\right)$. The test for $r_{1}=3$ and $r_{2}=5$, that is, $\widehat{\mu}_{35}$, is reported in Table 1 .

\subsection{Testing for Kurtosis}

Again, we derive the limiting distribution of the estimated kurtosis under arbitrary true $\kappa$ and then specialize it to $\kappa=3$ under normality. By Lemma 1 in Appendix, we have

Theorem 3 Suppose $X_{t}$ is stationary up to eighth order. Then

$$
\begin{aligned}
\sqrt{T}(\widehat{\kappa}-\kappa) & =\frac{\beta}{\widehat{\sigma}^{4}} \frac{1}{\sqrt{T}} \sum_{t=1}^{T} W_{t}+o_{p}(1), \\
\text { where } \beta & =\left[\begin{array}{ll}
1-4 \mu_{3}-2 \sigma^{2} \kappa
\end{array}\right], \quad W_{t}=\left[\begin{array}{c}
\left(X_{t}-\mu\right)^{4}-\mu_{4} \\
\left(X_{t}-\mu\right) \\
\left(X_{t}-\mu\right)^{2}-\sigma^{2}
\end{array}\right]
\end{aligned}
$$

and $\frac{1}{\sqrt{T}} \sum_{t=1}^{T} W_{t} \stackrel{d}{\longrightarrow} N(0, \Omega)$ with $\Omega=\lim _{T \rightarrow \infty} T E\left(\bar{W} \bar{W}^{\prime}\right)$. Let $\widehat{\sigma}^{2}$ and $\widehat{\Omega}$ be consistent estimates of $\sigma^{2}$ and $\Omega$, respectively. Then

$$
\widehat{\pi}_{4}(\kappa)=\frac{\sqrt{T}(\widehat{\kappa}-\kappa)}{s(\widehat{\kappa})} \stackrel{d}{\longrightarrow} N(0,1),
$$

where $s(\widehat{\kappa})=\left(\widehat{\beta} \widehat{\Omega} \widehat{\beta}^{\prime} / \widehat{\sigma}^{8}\right)^{1 / 2}$.

Note that the first component of $W_{t}$ depends on the fourth moment of $\left(X_{t}-\mu\right)^{4}$, which itself is a highly skewed random variable even if $X_{t}$ is not skewed. The convergence to normal could be extremely slow, and the sample estimate of $\widehat{\kappa}$ can deviate substantially from its true value even with a large number of observations. Thus for moderate sample sizes, the kurtosis test cannot be expected to be accurate. This will be confirmed by simulations in the next section. 


\subsection{Testing for Normality}

Under normality, $\tau=0$ and $\kappa=3$. Let $\widehat{\pi}_{3}$ be the test defined earlier for testing $\tau=0$, and let $\widehat{\pi}_{4}$ be the test statistic for kurtosis evaluated at $\kappa=3$. That is, $\widehat{\pi}_{4}=\widehat{\pi}_{4}(3)$. It can be shown that $\widehat{\pi}_{3}$ and $\widehat{\pi}_{4}$ are asymptotically independent under normality even for time series data. Thus a direct generalization of the Bera-Jarque test to dependent data is

$$
\widehat{\pi}_{34}=\widehat{\pi}_{3}^{2}+\widehat{\pi}_{4}^{2} \stackrel{d}{\longrightarrow} \chi_{2}^{2} .
$$

An asymptotically equivalent test, based directly on the third and fourth central moments, can be constructed as follows. Let

$$
Y_{T}=\left(\begin{array}{c}
\frac{1}{\sqrt{T}} \sum_{t=1}^{T}\left(X_{t}-\bar{X}\right)^{3} \\
\frac{1}{\sqrt{T}} \sum_{t=1}^{T}\left[\left(X_{t}-\bar{X}\right)^{4}-3\left(\widehat{\sigma}^{2}\right)^{2}\right]
\end{array}\right)
$$

Under normality, it can be shown that

$$
Y_{t}=\gamma \frac{1}{\sqrt{T}} \sum_{t=1}^{T} Z_{t}+o_{p}(1)
$$

where

$$
\gamma=\left[\begin{array}{cccc}
-3 \sigma^{2} & 0 & 1 & 0 \\
0 & -6 \sigma^{2} & 0 & 1
\end{array}\right], \quad Z_{t}=\left[\begin{array}{c}
\left(X_{t}-\mu\right) \\
\left(X_{t}-\mu\right)^{2}-\sigma^{2} \\
\left(X_{t}-\mu\right)^{3} \\
\left(X_{t}-\mu\right)^{4}-3 \sigma^{4}
\end{array}\right]
$$

with $\frac{1}{\sqrt{T}} \sum_{t=1}^{T} Z_{t} \stackrel{d}{\longrightarrow} N(0, \Phi)$ and $\Phi=\lim _{T \rightarrow \infty} T E\left(\bar{Z} \bar{Z}^{\prime}\right)$. Thus $Y_{T} \stackrel{d}{\longrightarrow} N\left(0, \gamma \Phi \gamma^{\prime}\right)$. Let $\widehat{\gamma}$ and $\widehat{\Phi}$ be consistent estimators of $\gamma$ and $\Phi$, respectively, we have

$$
\widehat{\mu}_{34}=Y_{T}^{\prime}\left(\widehat{\gamma} \widehat{\Phi} \widehat{\gamma}^{\prime}\right)^{-1} Y_{T} \stackrel{d}{\longrightarrow} \chi_{2}^{2} .
$$

Summarizing the above results, we have

Theorem 4 Suppose that $X_{t}$ is stationary. Then under the null hypothesis of normality,

$$
\begin{aligned}
& \widehat{\pi}_{34} \stackrel{d}{\longrightarrow} \chi_{2}^{2}, \\
& \widehat{\mu}_{34} \stackrel{d}{\longrightarrow} \chi_{2}^{2} .
\end{aligned}
$$




\subsection{Simulations}

To assess the size and power of the tests, we consider well-known distributions such as the normal, the $t$ and the $\chi^{2}$, as well as distributions from the generalized lambda family. This family encompasses a range of symmetric and asymmetric distributions that can be easily generated since they are defined in terms of the inverse of the cumulative distribution $F^{-1}(u)=\lambda_{1}+\left[u^{\lambda_{3}}-(1-u)^{\lambda_{4}}\right] / \lambda_{2}$, $0<u<1$. The $\lambda$ parameters are taken from Table 1 of Randles, Fligner, Policello and Wolfe (1980). Specifically, data are generated from 7 symmetric and 8 skewed distributions:

$\mathrm{S} 1: N(0,1)$

$\mathrm{S} 2: t_{5}$

$\mathrm{S} 3: e_{1} I(z \leq .5)+e_{2} I(z>.5)$, where $z \sim U(0,1), e_{1} \sim N(-1,1)$, and $e_{2} \sim N(1,1)$;

$\mathrm{S} 4: F^{-1}(u)=\lambda_{1}+\left[u^{\lambda_{3}}-(1-u)^{\lambda_{4}}\right] / \lambda_{2}, \lambda_{1}=0, \lambda_{2}=.19754, \lambda_{3}=.134915, \lambda_{4}=.134915$;

S5 : $F^{-1}(u)=\lambda_{1}+\left[u^{\lambda_{3}}-(1-u)^{\lambda_{4}}\right] / \lambda_{2}, \lambda_{1}=0, \lambda_{2}=-1, \lambda_{3}=-.08, \lambda_{4}=-.08 ;$

S6 $: F^{-1}(u)=\lambda_{1}+\left[u^{\lambda_{3}}-(1-u)^{\lambda_{4}}\right] / \lambda_{2}, \lambda_{1}=0, \lambda_{2}=-.397912, \lambda_{3}=-.16, \lambda_{4}=-.16$;

S7 $: F^{-1}(u)=\lambda_{1}+\left[u^{\lambda_{3}}-(1-u)^{\lambda_{4}}\right] / \lambda_{2}, \lambda_{1}=0, \lambda_{2}=-1, \lambda_{3}=-.24, \lambda_{4}=-.24$.

A1 : lognormal: $\exp (e), e \sim N(0,1)$;

A2 : $\chi_{2}^{2}$;

A3 : exponential: $-\ln (e), e \sim N(0,1)$,

A4 $: F^{-1}(u)=\lambda_{1}+\left[u^{\lambda_{3}}-(1-u)^{\lambda_{4}}\right] / \lambda_{2}, \lambda_{1}=0, \lambda_{2}=1.0, \lambda_{3}=1.4, \lambda_{4}=.25$;

A5 $: F^{-1}(u)=\lambda_{1}+\left[u^{\lambda_{3}}-(1-u)^{\lambda_{4}}\right] / \lambda_{2}, \lambda_{1}=0, \lambda_{2}=-1, \lambda_{3}=-.0075, \lambda_{4}=-.03$;

A6 : $F^{-1}(u)=\lambda_{1}+\left[u^{\lambda_{3}}-(1-u)^{\lambda_{4}}\right] / \lambda_{2}, \lambda_{1}=0, \lambda_{2}=-1, \lambda_{3}=-.1, \lambda_{4}=-.18 ;$

A7 : $F^{-1}(u)=\lambda_{1}+\left[u^{\lambda_{3}}-(1-u)^{\lambda_{4}}\right] / \lambda_{2}, \lambda_{1}=0, \lambda_{2}=-1, \lambda_{3}=-.001, \lambda_{4}=-.13$;

A8 $: F^{-1}(u)=\lambda_{1}+\left[u^{\lambda_{3}}-(1-u)^{\lambda_{4}}\right] / \lambda_{2}, \lambda_{1}=0, \lambda_{2}=-1, \lambda_{3}=-.0001, \lambda_{4}=-.17$.

To evaluate the size of the test for skewness, we draw $e_{t}$ from seven symmetric distributions. The power of the tests is assessed by considering eight asymmetric distributions. Since the kurtosis of all fifteen distributions are known (and are given in Table 2), the size and power of $\widehat{\pi}_{4}$ as well as the normality tests can be easily obtained.

The data used in the simulations are generated as $X_{t}=\rho X_{t-1}+e_{t}$. Many values of $\rho$ were considered, and results are presented for $\rho=0, .5$ and .8. The long run covariance matrix is 
estimated by kernel method with the truncation lag selected using the automatic procedure with prewhitening as discussed in Andrews and Monahan (1992). We use the Parzen window:

$$
\begin{aligned}
w(x) & =1-6 x^{2}+6|x|^{3} \text { if } 0 \leq|x| \leq 1 / 2 \\
& =2(1-|x|)^{3} \text { if } 1 / 2 \leq|x| \leq 1 .
\end{aligned}
$$

\subsection{Skewness}

We consider both one and two tailed tests for symmetry (denoted $\widehat{\pi}_{3}^{*}$ and $\widehat{\pi}_{3}^{* *}$, respectively). Results are reported at the $5 \%$ level without loss of generality. The critical values are 1.64 (one tailed) and 1.96 (two tailed). We also consider $\widehat{\mu}_{35}$, a joint test of the third and fifth central moment, and the $5 \%$ critical value is 5.99. Three sample sizes are considered: $T=100,200$, and 500 . Table 1 indicates that $\widehat{\pi}_{3}$ has accurate size even for small $T$, but the $\widehat{\mu}_{35}$ statistic rejects less often than $\pi_{3}$ under the null. The size of the tests are, however, quite robust to the degree of persistence in the data. Note also that although the $t_{5}$ distribution does not have finite 6 th moment, testing symmetry still gives very good size.

The two-sided test has low power, but imposing a priori information on the direction of skewness leads to substantial power gains. All the tests considered have low power for A4, A5 and A6 unless the sample size is large (say, more than 200 observations). The statistics developed in Randles et al. (1980) for testing symmetry in i.i.d. data also have low power for the same distributions, all of which have large kurtosis. In general, $\widehat{\mu}_{35}$ has very good power even for $T$ as small as 50 . However, whereas the size of the test is quite robust to serial correlation in the data, the power function is quite sensitive to persistence. Results for $\rho=.8$ reveal that the power of the tests drop significantly

when the degree of persistence increases. The reason is that for $\operatorname{AR}(1)$ models, $y_{t}=\sum_{j=1}^{t} \alpha^{j} u_{t-j}$ (assuming $\left.y_{0}=0\right)$. In the limit when $\alpha=1, y_{t}(\operatorname{scaled}$ by $\sqrt{T})$ is asymptotically normal. The data thus becomes more symmetric as persistence increases. This is confirmed upon comparison with results for i.i.d. data, which is given in panel (a) of Table 1.

From the simulations, $\widehat{\mu}_{35}$ has a smaller probability of Type I error and higher power and dominates $\widehat{\pi}_{3}$. Thus this test is recommended when symmetry is the main concern. In principle, a joint test of more moments is feasible, and there is no reason to stop at testing two moments jointly. We have laid out the general framework in which more comprehensive tests can be developed. A practical strategy would be to start with the third moment and consider joint tests of higher moments only if one does not reject the null hypothesis. 


\subsection{Kurtosis and Normality}

In Table 2 , we report results for $\widehat{\pi}_{4}(\kappa)$, which tests the true population value of $\kappa$, and $\widehat{\pi}_{4}(3)$, which tests $\kappa=3$ as would be the case under normality. There are two notable results. First, there are large size distortions, so that a large Type 1 error can be expected if one was to test $\kappa=\kappa^{0}$. Second, while the test has power to reject $\kappa=3$, the power is very low for the sample sizes considered. In many cases, serial correlation in the data further reduces the power of the test. For example, consider case A3 with $\kappa=9$. With $T=1000$, the power is .89 when $\rho=0$, falls to .84 when $\rho=.5$, and is only .24 when $\rho=.8$. One needs more than 5000 observations to reject the hypothesis that $\kappa=3$ when $\rho=.8$. For this reason, we report results for sample sizes much larger than for $\widehat{\tau}$ to highlight the problems with testing for kurtosis in finite samples.

To understand the properties of the kurtosis tests, we report in Table 3 the average estimates of $\kappa$ and $\tau$ at the different sample sizes. Three results are noteworthy. First, both $\widehat{\tau}$ and $\kappa$ are generally downward biased with biases that are increasing in $\rho$. However, the biases are substantially larger for $\widehat{\kappa}$. Second, even with $T$ as large as $5000, \kappa$ cannot be precisely estimated from serially correlated data. In some cases, $\widehat{\kappa}$ is severely biased even for iid data (see, for example, case A1). This result has the important empirical implication that the sample kurtosis measure is generally unreliable and should always be viewed with caution. Third, the one exception when $\widehat{\kappa}$ can be well estimated is when $\kappa=3$. This is important in interpreting the results for testing normality.

Results for testing normality are reported in Table 4. Except for the first row which is based on the normal distribution and thus indicates size, all other rows indicate power. The $\widehat{\mu}_{34}$ test is generally more powerful than the $\widehat{\pi}_{34}$ test. Since the kurtosis test has such low power, the results for normality by and large reflect the results for the tests for skewness. The tests have low power when a distribution is symmetric.

The fact that the kurtosis test has large size distortions might appear problematic for the normality test. Interestingly, however, this is not the case. This is because $\widehat{\kappa}$ precisely estimates $\kappa$ when $\kappa=3$ and size distortion is not an issue. Thus, while the kurtosis test is itself not very useful per se, we can still use it to test normality, as in Bera and Jarque (1981).

\section{Empirical Applications}

The tests are applied to 21 macroeconomic time series. Data for GDP, the GDP deflator, the consumption of durables, final sales, the consumption of non-durables, residential investment, and non-residential investment are taken from the national accounts and are quarterly data. The unemployment rate, employment, M2, CPI are monthly series. The 30 day interest rate, and M2 
are weekly data. ${ }^{1}$ With the exception of the interest rate and the unemployment rate (which we do not take logs), we take first difference of the logarithm of the data before applying the tests. We also considered three exchange rates (in logged first differences), and the value as well as the equally weighted CRSP daily stock returns. These data are not transformed. The sample skewness and kurtosis coefficients for the 21 series are also reported, along with tests for skewness, kurtosis, and normality.

The first column of Table 4 reports tests for symmetry, i,e.testing $\tau=0$, with $\widehat{\tau}$ given in column 4. Several aspects of the results are of note. As in Delong and Summers (1985), we fail to reject symmetry in output and industrial production. However, while these authors find asymmetry in the unemployment rate, using a longer sample period and a different procedure to conduct inference, we find no evidence of skewness in the unemployment rate. The US-Japan exchange rate, CPI inflation, as well as stock returns reject symmetry at $1 \%$ level. We also reject symmetry in manufacturing employment and the consumption of durable goods at the $10 \%$ level. Interesting, series that exhibit skewness also failed the conditional symmetry test of Bai and $\mathrm{Ng}(2001)$.

The second column of Table 4 reports results for testing $\kappa=3$ with $\widehat{\kappa}$ given in column 5 . We failed to find evidence of excess kurtosis in any of the real variables but found evidence of fat-tailed behavior in US-Japan exchange rate, the 30 day interest rate, and the two stock returns. These are financial series whose fat tailed properties have been well-documented. Our evidence is especially convincing in view of the lower power of the test for kurtosis reported earlier.

Results for the normality test is reported in the third column of Table 4 . We reject normality in the US-Japan exchange rate, the unemployment rate, the CPI inflation, the 30 day interest rate, and the two stock return series. With the exception of the 30 day interest rate which failed the kurtosis test but has no evidence of skewness, the remaining series that exhibit non-Guassian behavior failed the symmetry test. This accords with our observation that the power of the normality test is derived from asymmetry.

\section{Concluding Comments}

The goal of this paper is to obtain tests for skewness, kurtosis, and a joint test of normality suited for time series observations. Monte Carlo simulations accord with our prior that tests for kurtosis will have low power because of the high moments involved. In finite samples, the test for kurtosis has poor size. The difficulty in estimating kurtosis does not pose a size problem for normality tests, however. Combining the coefficient of skewness with kurtosis as in Bera and Jarque is still

\footnotetext{
${ }^{1}$ All data are taken from the Economic Time Series Page, and URL is: vos.business.uab.edu/data.htm.
} 
useful for time series data, once the limiting variance takes into account of serial correlation in the data. Nonetheless, the primary source of power in the test for normality is derived from the test for skewness.

This paper has focused on the unconditional moment properties, in contrast to the conditional distributions as in Bai and $\mathrm{Ng}$ (2001). To test for conditional symmetry, a model of the data generating process is required, and the tests are applied to the regression residuals. In contrast, no assumption about the data generating process is necessary for testing the unconditional moments of the data. The results of the present analysis only depends on stationarity and the existence of some moments. Furthermore, the unconditional tests are applied to the observed data rather than to the regression residuals. In cases such as analyzed in Delong and Summers (1985) when skewness of GDP is the object of interest, the unconditional tests developed in this paper are more appropriate. Cabellero (1992) emphasized the role that asymmetric shocks might play in explaining why firms' job creation policies might be different from job destruction policies. In such a case, testing for conditional symmetry in gross job flows may be more appropriate.

It should also clear that unconditional symmetry and conditional symmetry generally do not imply each other. Consider an extreme example, $X_{t}=\epsilon_{t}-\epsilon_{t-1}$, where $\epsilon_{t}$ are independent and identically distributed (i.i.d.). Whether or not $\epsilon_{t}$ has a symmetric distribution, $X_{t}$ is always symmetric because $X_{t}$ and $-X_{t}$ have the same distribution. However, conditional on the information at $t-1$ (which include $\epsilon_{t-1}$ ), the conditional distribution of $X_{t}$ will be asymmetric provided $\epsilon_{t}$ is asymmetric. 


\section{Appendix}

The following lemma is used for Theorem 1.

Lemma 1 Suppose $X_{t}$ is stationary up to order $r$ for some $r \geq 2$. Then

$$
\widehat{\mu}_{r}=\frac{1}{\sqrt{T}} \sum_{t=1}^{T}\left(X_{t}-\bar{X}\right)^{r}=\frac{1}{\sqrt{T}} \sum_{t=1}^{T}\left(X_{t}-\mu\right)^{r}-r \mu_{r-1} \frac{1}{\sqrt{T}} \sum_{t=1}^{T}\left(X_{t}-\mu\right)+o_{p}(1) .
$$

Furthermore, by the delta method,

$$
\sqrt{T}\left(\left(\widehat{\sigma}^{2}\right)^{r / 2}-\left(\sigma^{2}\right)^{r / 2}\right)=\frac{r}{2}\left(\sigma^{2}\right)^{r / 2-1}\left[\widehat{\sigma}^{2}-\sigma^{2}\right]+o_{p}(1) .
$$

Proof: We show (without loss of generality) the derivations for $r=3$. The generalization is immediate.

$$
\begin{aligned}
\frac{1}{\sqrt{T}} \sum_{t=1}^{T}\left(X_{t}-\bar{X}\right)^{3}= & \frac{1}{\sqrt{T}} \sum_{t=1}^{T}\left(X_{t}-\mu+\mu-\bar{X}\right)^{3} \\
= & \frac{1}{\sqrt{T}} \sum_{t=1}^{T}\left(X_{t}-\mu\right)^{3}+3 \frac{1}{T} \sum_{t=1}^{T}\left(X_{t}-\mu\right)^{2} \sqrt{T}(\mu-\bar{X}) \\
& +3(\mu-\bar{X})^{2} \frac{1}{\sqrt{T}} \sum_{t=1}^{T}\left(X_{t}-\mu\right)+\sqrt{T}(\mu-\bar{X})^{3}
\end{aligned}
$$

The last two terms are $o_{p}(1)$ since $\sqrt{T}(\bar{X}-\mu)=O_{p}(1)$. Finally note that $\left.\frac{1}{T} \sum_{t=1}^{T}\left(X_{t}-\mu\right)^{2}\right)=$ $\mu_{2}+o_{p}(1)$.

\section{Proof of Theorem 1.}

$$
\begin{aligned}
\widehat{\tau}-\tau= & \frac{\widehat{\mu}_{3}}{\widehat{\sigma}^{3}}-\frac{\mu_{3}}{\sigma^{3}}=\frac{\widehat{\mu}_{3}-\mu_{3}}{\widehat{\sigma}^{3}}-\tau \frac{\widehat{\sigma}^{3}-\sigma^{3}}{\widehat{\sigma}^{3}} \\
= & {\left[\frac{\frac{1}{T} \sum_{t=1}^{T}\left(X_{t}-\bar{X}\right)^{3}-\mu_{3}}{\widehat{\sigma}^{3}}\right]-\tau\left[\frac{\left(\widehat{\sigma}^{2}\right)^{3 / 2}-\left(\sigma^{2}\right)^{3 / 2}}{\widehat{\sigma}^{3}}\right] } \\
= & \frac{1}{\widehat{\sigma}^{3}}\left[\frac{1}{T} \sum_{t=1}^{T}\left(\left(X_{t}-\mu\right)^{3}-\mu_{3}\right)-3\left(\frac{1}{T} \sum_{t=1}^{T}\left(X_{t}-\mu\right)^{2}\right)(\bar{X}-\mu)\right]-\frac{3 \tau \sigma\left(\widehat{\sigma}^{2}-\sigma^{2}\right)}{2 \widehat{\sigma}^{3}}+o_{p}(1) \\
= & \frac{1}{\widehat{\sigma}^{3}}\left[\frac{1}{T} \sum_{t=1}^{T}\left(\left(X_{t}-\mu\right)^{3}-\mu_{3}\right)-3\left(\frac{1}{T} \sum_{t=1}^{T}\left(X_{t}-\mu\right)^{2}\right)\left(\frac{1}{T} \sum_{t=1}^{T} X_{t}-\mu\right)\right] \\
& -\frac{3 \tau \sigma}{2 T \widehat{\sigma}^{3}}\left[\sum_{t=1}^{T}\left(X_{t}-\mu\right)^{2}-\sigma^{2}\right]+o_{p}(1) \\
= & \frac{1}{\widehat{\sigma}^{3}}\left[1-3 \sigma^{2}-\frac{3 \sigma \tau}{2}\right] \frac{1}{T}\left[\begin{array}{c}
\sum_{t=1}^{T}\left[\left(X_{t}-\mu\right)^{3}-\mu_{3}\right] \\
\sum_{t=1}^{T}\left(X_{t}-\mu\right) \\
\sum_{t=1}^{T}\left[\left(X_{t}-\mu\right)^{2}-\sigma^{2}\right]
\end{array}\right]+o_{p}(1) \\
\equiv & \frac{\alpha}{\widehat{\sigma}^{3}} \frac{1}{T} \sum_{t=1}^{T} Z_{t}+o_{p}(1)
\end{aligned}
$$




\section{References}

Andrews, D. W. K. and Monahan, J. (1992), An Improved Heteroskedasticity and Autocorrelation Consistent Covariance Matrix Estimator, Econometrica 60, 953-966.

Bai, J. and Ng, S. (2001), A Test for Conditional Symmetry in Time Series Models, Journal of Econometrics p. Forthcoming.

Bera, A. and Jarque, C. (1981), Efficient Tests for Normality, Heteroskedasticty, and Serial Independence of Regression Residuals: Monte Carlo Evidence, Economics Letter 7, 313-318.

Cabellero, R. J. (1992), A Fallacy of Composition, American Economic Review 82:5, 1279-1292.

Delong, J. B. and Summers, L. H. (1985), Are Business Cycle Symmetrical, American Business Cycle: Continuity and Change, University of Chicago Press, Chicago.

Kendall, M. and Stuart, A. (1969), The Advanced Theory of Statistics, Mcgraw Hill.

Randles, R. H., Fligner, M. A., Policello, G. E. and Wolfe, D. A. (1980), An Asymptotically Distrbution-Free Test for Symmetry Versus Asymmetry, Journal of the American Statistical Association 75, 168-172. 
Table 1: Size and Power of the Test Symmetry for $\tau=0$ :

\begin{tabular}{|c|c|c|c|c|c|c|c|c|c|c|c|}
\hline \multicolumn{3}{|c|}{$\rho=.0$} & \multicolumn{3}{|c|}{$\mathrm{T}=100$} & \multicolumn{3}{|c|}{$\mathrm{T}=200$} & \multicolumn{3}{|c|}{$\mathrm{T}=500$} \\
\hline & $\tau$ & $\kappa$ & $\widehat{\pi}_{3}^{* *}$ & $\widehat{\pi}_{3}^{*}$ & $\widehat{\mu}_{35}$ & $\widehat{\pi}_{3}^{* *}$ & $\widehat{\pi}_{3}^{*}$ & $\widehat{\mu}_{35}$ & $\widehat{\pi}_{3}^{* *}$ & $\widehat{\pi}_{3}^{*}$ & $\widehat{\mu}_{35}$ \\
\hline$\overline{\text { S1 }}$ & 0.0 & 3.0 & 0.04 & 0.05 & 0.03 & 0.05 & 0.06 & 0.04 & 0.06 & 0.06 & 0.04 \\
\hline S2 & 0.0 & 9.0 & 0.03 & 0.05 & 0.02 & 0.04 & 0.05 & 0.03 & 0.03 & 0.04 & 0.02 \\
\hline S3 & 0.0 & 2.5 & 0.04 & 0.05 & 0.03 & 0.05 & 0.06 & 0.04 & 0.05 & 0.06 & 0.04 \\
\hline $\mathrm{S} 4$ & 0.0 & 3.0 & 0.03 & 0.04 & 0.02 & 0.04 & 0.05 & 0.03 & 0.05 & 0.05 & 0.04 \\
\hline S5 & 0.0 & 6.0 & 0.03 & 0.04 & 0.02 & 0.04 & 0.05 & 0.02 & 0.04 & 0.06 & 0.03 \\
\hline S6 & 0.0 & 11.6 & 0.02 & 0.04 & 0.02 & 0.04 & 0.04 & 0.02 & 0.03 & 0.04 & 0.03 \\
\hline S7 & 0.0 & 126.0 & 0.03 & 0.05 & 0.02 & 0.02 & 0.04 & 0.02 & 0.03 & 0.04 & 0.02 \\
\hline$\overline{\mathrm{A} 1}$ & 6.18 & 113.9 & 0.42 & 0.62 & 0.59 & 0.51 & 0.68 & 0.80 & 0.68 & 0.81 & 0.95 \\
\hline $\mathrm{A} 2$ & 2.0 & 9.0 & 0.72 & 0.87 & 0.93 & 0.90 & 0.96 & 1.00 & 0.98 & 0.99 & 1.00 \\
\hline A3 & 2.0 & 9.0 & 0.74 & 0.88 & 0.95 & 0.90 & 0.97 & 1.00 & 0.98 & 0.99 & 1.00 \\
\hline $\mathrm{A} 4$ & .5 & 2.2 & 0.84 & 0.92 & 0.63 & 1.00 & 1.00 & 0.99 & 1.00 & 1.00 & 1.00 \\
\hline A5 & 1.5 & 7.5 & 0.65 & 0.84 & 0.78 & 0.85 & 0.95 & 0.99 & 0.98 & 1.00 & 1.00 \\
\hline $\mathrm{A} 6$ & 2.0 & 21.2 & 0.23 & 0.41 & 0.21 & 0.43 & 0.64 & 0.54 & 0.72 & 0.85 & 0.93 \\
\hline $\mathrm{A} 7$ & 3.16 & 23.8 & 0.56 & 0.76 & 0.78 & 0.74 & 0.87 & 0.95 & 0.87 & 0.94 & 1.00 \\
\hline A8 & 3.8 & 40.7 & 0.52 & 0.71 & 0.73 & 0.67 & 0.82 & 0.94 & 0.83 & 0.92 & 0.99 \\
\hline \multicolumn{3}{|c|}{$\rho=.5$} & & & & & & & & & \\
\hline S1 & 0.0 & 3.0 & 0.04 & 0.05 & 0.03 & 0.03 & 0.04 & 0.02 & 0.04 & 0.05 & 0.03 \\
\hline $\mathrm{S} 2$ & 0.0 & 9.0 & 0.04 & 0.05 & 0.02 & 0.03 & 0.04 & 0.03 & 0.04 & 0.04 & 0.03 \\
\hline S3 & 0.0 & 2.5 & 0.03 & 0.04 & 0.02 & 0.03 & 0.05 & 0.03 & 0.04 & 0.05 & 0.03 \\
\hline $\mathrm{S} 4$ & 0.0 & 3.0 & 0.03 & 0.04 & 0.02 & 0.03 & 0.04 & 0.03 & 0.05 & 0.05 & 0.04 \\
\hline S5 & 0.0 & 6.0 & 0.03 & 0.04 & 0.03 & 0.04 & 0.06 & 0.03 & 0.05 & 0.06 & 0.03 \\
\hline S6 & 0.0 & 11.6 & 0.04 & 0.04 & 0.02 & 0.04 & 0.04 & 0.02 & 0.04 & 0.05 & 0.03 \\
\hline S7 & 0.0 & 126.0 & 0.04 & 0.04 & 0.02 & 0.03 & 0.04 & 0.03 & 0.03 & 0.04 & 0.03 \\
\hline $\mathrm{A} 1$ & 6.18 & 113.9 & 0.45 & 0.64 & 0.58 & 0.51 & 0.70 & 0.77 & 0.66 & 0.80 & 0.94 \\
\hline $\mathrm{A} 2$ & 2.0 & 9.0 & 0.61 & 0.79 & 0.73 & 0.85 & 0.93 & 0.98 & 0.97 & 0.99 & 1.00 \\
\hline A3 & 2.0 & 9.0 & 0.64 & 0.84 & 0.74 & 0.84 & 0.94 & 0.98 & 0.96 & 0.98 & 1.00 \\
\hline$\overline{\mathrm{A} 4}$ & .5 & 2.2 & 0.32 & 0.50 & 0.21 & 0.69 & 0.83 & 0.53 & 0.99 & 1.00 & 0.97 \\
\hline A5 & 1.5 & 7.5 & 0.49 & 0.72 & 0.44 & 0.76 & 0.89 & 0.89 & 0.96 & 0.99 & 1.00 \\
\hline$\overline{\mathrm{A} 6}$ & 2.0 & 21.2 & 0.21 & 0.38 & 0.18 & 0.39 & 0.55 & 0.39 & 0.68 & 0.81 & 0.83 \\
\hline A7 & 3.16 & 23.8 & 0.55 & 0.72 & 0.68 & 0.70 & 0.84 & 0.92 & 0.85 & 0.93 & 0.99 \\
\hline A8 & 3.8 & 40.7 & 0.51 & 0.68 & 0.66 & 0.66 & 0.80 & 0.91 & 0.82 & 0.91 & 0.99 \\
\hline \multicolumn{3}{|c|}{$\rho=.8$} & & & & & & & & & \\
\hline S1 & 0.0 & 3.0 & 0.01 & 0.03 & 0.01 & 0.03 & 0.03 & 0.02 & 0.03 & 0.04 & 0.02 \\
\hline $\mathrm{S} 2$ & 0.0 & 9.0 & 0.02 & 0.04 & 0.01 & 0.03 & 0.05 & 0.01 & 0.03 & 0.04 & 0.03 \\
\hline S3 & 0.0 & 2.5 & 0.02 & 0.03 & 0.02 & 0.01 & 0.03 & 0.01 & 0.04 & 0.05 & 0.03 \\
\hline $\mathrm{S} 4$ & 0.0 & 3.0 & 0.01 & 0.02 & 0.01 & 0.03 & 0.03 & 0.02 & 0.04 & 0.04 & 0.03 \\
\hline S5 & 0.0 & 6.0 & 0.02 & 0.03 & 0.01 & 0.03 & 0.04 & 0.01 & 0.04 & 0.04 & 0.02 \\
\hline S6 & 0.0 & 11.6 & 0.03 & 0.04 & 0.02 & 0.03 & 0.03 & 0.02 & 0.04 & 0.05 & 0.02 \\
\hline S7 & 0.0 & 126.0 & 0.03 & 0.04 & 0.02 & 0.03 & 0.04 & 0.01 & 0.03 & 0.04 & 0.02 \\
\hline A1 & 6.18 & 113.9 & 0.10 & 0.21 & 0.10 & 0.30 & 0.50 & 0.25 & 0.59 & 0.75 & 0.80 \\
\hline $\mathrm{A} 2$ & 2.0 & 9.0 & 0.03 & 0.05 & 0.12 & 0.13 & 0.28 & 0.18 & 0.70 & 0.86 & 0.73 \\
\hline A3 & 2.0 & 9.0 & 0.04 & 0.05 & 0.11 & 0.13 & 0.25 & 0.16 & 0.71 & 0.85 & 0.75 \\
\hline A4 & .5 & 2.2 & 0.10 & 0.19 & 0.08 & 0.12 & 0.24 & 0.08 & 0.29 & 0.44 & 0.15 \\
\hline$\overline{\mathrm{A} 5}$ & 1.5 & 7.5 & 0.05 & 0.15 & 0.03 & 0.21 & 0.40 & 0.10 & 0.61 & 0.79 & 0.53 \\
\hline $\mathrm{A} 6$ & 2.0 & 21.2 & 0.08 & 0.19 & 0.04 & 0.14 & 0.28 & 0.09 & 0.41 & 0.61 & 0.34 \\
\hline A7 & 3.16 & 23.8 & 0.06 & 0.14 & 0.08 & 0.24 & 0.45 & 0.19 & 0.70 & 0.84 & 0.87 \\
\hline A8 & 3.8 & 40.7 & 0.06 & 0.17 & 0.07 & 0.29 & 0.50 & 0.22 & 0.70 & 0.84 & 0.88 \\
\hline
\end{tabular}

** denote two sided test. ${ }^{*}$ denote one sided test. 
Table 2: Size and Power of the Test Kurtosis

\begin{tabular}{|c|c|c|c|c|c|c|c|c|c|c|c|c|c|c|}
\hline \multicolumn{3}{|c|}{$\rho=.0$} & \multicolumn{2}{|c|}{$\mathrm{T}=100$} & \multicolumn{2}{|c|}{$\mathrm{T}=200$} & \multicolumn{2}{|c|}{$\mathrm{T}=500$} & \multicolumn{2}{|c|}{$\mathrm{T}=1000$} & \multicolumn{2}{|c|}{$\mathrm{T}=2500$} & \multicolumn{2}{|c|}{$\mathrm{T}=5000$} \\
\hline & $\tau$ & $\kappa$ & $\widehat{\pi}_{4}(\kappa)$ & $\widehat{\pi}_{4}(3)$ & $\widehat{\pi}_{4}(\kappa)$ & $\widehat{\pi}_{4}(3)$ & $\widehat{\pi}_{4}(\kappa)$ & $\widehat{\pi}_{4}(3)$ & $\widehat{\pi}_{4}(\kappa)$ & $\widehat{\pi}_{4}(3)$ & $\widehat{\pi}_{4}(\kappa)$ & $\widehat{\pi}_{4}(3)$ & $\widehat{\pi}_{4}(\kappa)$ & $\widehat{\pi}_{4}(3)$ \\
\hline S1 & 0.0 & 3.0 & 0.02 & 0.02 & 0.02 & 0.02 & 0.02 & 0.02 & 0.02 & 0.02 & 0.01 & 0.01 & 0.02 & 0.02 \\
\hline S2 & 0.0 & 9.0 & 0.66 & 0.00 & 0.73 & 0.10 & 0.69 & 0.51 & 0.64 & 0.70 & 0.61 & 0.82 & 0.58 & 0.88 \\
\hline S3 & 0.0 & 2.5 & 0.01 & 0.20 & 0.00 & 0.41 & 0.01 & 0.80 & 0.01 & 0.97 & 0.00 & 1.00 & 0.00 & 1.00 \\
\hline S4 & 0.0 & 3.0 & 0.02 & 0.02 & 0.01 & 0.01 & 0.01 & 0.01 & 0.01 & 0.01 & 0.01 & 0.01 & 0.02 & 0.02 \\
\hline S5 & 0.0 & 6.0 & 0.32 & 0.01 & 0.36 & 0.14 & 0.37 & 0.65 & 0.32 & 0.86 & 0.24 & 0.94 & 0.23 & 0.99 \\
\hline S6 & 0.0 & 11.6 & 0.59 & 0.03 & 0.69 & 0.23 & 0.66 & 0.63 & 0.58 & 0.72 & 0.53 & 0.84 & 0.46 & 0.89 \\
\hline S7 & 0.0 & 126.0 & 0.42 & 0.05 & 0.47 & 0.25 & 0.36 & 0.50 & 0.25 & 0.58 & 0.11 & 0.67 & 0.05 & 0.70 \\
\hline $\mathrm{A} 1$ & 6.18 & 113.9 & 0.17 & 0.14 & 0.32 & 0.26 & 0.70 & 0.40 & 0.85 & 0.49 & 0.78 & 0.60 & 0.75 & 0.67 \\
\hline $\mathrm{A} 2$ & 2.0 & 9.0 & 0.22 & 0.07 & 0.28 & 0.35 & 0.29 & 0.75 & 0.24 & 0.92 & 0.17 & 0.98 & 0.15 & 1.00 \\
\hline A3 & 2.0 & 9.0 & 0.24 & 0.07 & 0.27 & 0.37 & 0.27 & 0.73 & 0.25 & 0.89 & 0.16 & 0.97 & 0.14 & 1.00 \\
\hline $\mathrm{A} 4$ & .5 & 2.2 & 0.01 & 0.50 & 0.01 & 0.80 & 0.01 & 0.99 & 0.01 & 1.00 & 0.01 & 1.00 & 0.02 & 1.00 \\
\hline $\mathrm{A} 5$ & 1.5 & 7.5 & 0.34 & 0.03 & 0.36 & 0.18 & 0.34 & 0.65 & 0.31 & 0.84 & 0.23 & 0.97 & 0.17 & 0.99 \\
\hline $\mathrm{A} 6$ & 2.0 & 21.2 & 0.61 & 0.04 & 0.80 & 0.24 & 0.77 & 0.51 & 0.74 & 0.63 & 0.67 & 0.75 & 0.62 & 0.81 \\
\hline A7 & 3.16 & 23.8 & 0.33 & 0.14 & 0.57 & 0.33 & 0.66 & 0.58 & 0.58 & 0.66 & 0.51 & 0.82 & 0.46 & 0.88 \\
\hline A8 & 3.8 & 40.7 & 0.29 & 0.14 & 0.57 & 0.33 & 0.78 & 0.50 & 0.73 & 0.60 & 0.67 & 0.75 & 0.61 & 0.79 \\
\hline \multicolumn{3}{|c|}{$\rho=.5$} & & & & & & & & & & & & \\
\hline S1 & 0.0 & 3.0 & 0.01 & 0.01 & 0.01 & 0.01 & 0.02 & 0.02 & 0.03 & 0.03 & 0.03 & 0.03 & 0.01 & 0.01 \\
\hline S2 & 0.0 & 9.0 & 0.57 & 0.01 & 0.83 & 0.04 & 0.85 & 0.28 & 0.82 & 0.58 & 0.84 & 0.80 & 0.82 & 0.86 \\
\hline S3 & 0.0 & 2.5 & 0.00 & 0.06 & 0.00 & 0.11 & 0.02 & 0.32 & 0.12 & 0.51 & 0.54 & 0.86 & 0.91 & 0.99 \\
\hline $\mathrm{S} 4$ & 0.0 & 3.0 & 0.01 & 0.01 & 0.02 & 0.02 & 0.02 & 0.02 & 0.02 & 0.02 & 0.02 & 0.02 & 0.01 & 0.01 \\
\hline S5 & 0.0 & 6.0 & 0.33 & 0.01 & 0.56 & 0.05 & 0.66 & 0.34 & 0.68 & 0.74 & 0.72 & 0.92 & 0.81 & 0.98 \\
\hline S6 & 0.0 & 11.6 & 0.51 & 0.03 & 0.79 & 0.10 & 0.83 & 0.48 & 0.80 & 0.68 & 0.80 & 0.83 & 0.79 & 0.88 \\
\hline S7 & 0.0 & 126.0 & 0.36 & 0.06 & 0.56 & 0.15 & 0.60 & 0.45 & 0.54 & 0.56 & 0.38 & 0.66 & 0.29 & 0.69 \\
\hline $\mathrm{A} 1$ & 6.18 & 113.9 & 0.22 & 0.15 & 0.33 & 0.27 & 0.66 & 0.40 & 0.88 & 0.48 & 0.91 & 0.59 & 0.88 & 0.67 \\
\hline $\mathrm{A} 2$ & 2.0 & 9.0 & 0.36 & 0.04 & 0.52 & 0.18 & 0.68 & 0.63 & 0.72 & 0.87 & 0.79 & 0.97 & 0.86 & 0.99 \\
\hline A3 & 2.0 & 9.0 & 0.34 & 0.04 & 0.51 & 0.18 & 0.63 & 0.60 & 0.72 & 0.84 & 0.79 & 0.96 & 0.86 & 0.99 \\
\hline $\mathrm{A} 4$ & .5 & 2.2 & 0.00 & 0.19 & 0.02 & 0.38 & 0.23 & 0.68 & 0.71 & 0.90 & 1.00 & 1.00 & 1.00 & 1.00 \\
\hline A5 & 1.5 & 7.5 & 0.40 & 0.02 & 0.55 & 0.07 & 0.67 & 0.44 & 0.69 & 0.75 & 0.76 & 0.96 & 0.80 & 0.98 \\
\hline A6 & 2.0 & 21.2 & 0.53 & 0.03 & 0.79 & 0.12 & 0.89 & 0.43 & 0.88 & 0.60 & 0.86 & 0.74 & 0.83 & 0.80 \\
\hline A7 & 3.16 & 23.8 & 0.34 & 0.08 & 0.59 & 0.26 & 0.83 & 0.52 & 0.81 & 0.63 & 0.81 & 0.80 & 0.79 & 0.88 \\
\hline A8 & 3.8 & 40.7 & 0.30 & 0.11 & 0.55 & 0.27 & 0.88 & 0.49 & 0.89 & 0.58 & 0.87 & 0.74 & 0.83 & 0.79 \\
\hline \multicolumn{3}{|c|}{$\rho=.8$} & & & & & & & & & & & & \\
\hline S1 & 0.0 & 3.0 & 0.01 & 0.01 & 0.01 & 0.01 & 0.02 & 0.02 & 0.02 & 0.02 & 0.03 & 0.03 & 0.02 & 0.02 \\
\hline S2 & 0.0 & 9.0 & 0.22 & 0.01 & 0.54 & 0.01 & 0.95 & 0.01 & 0.96 & 0.07 & 0.96 & 0.37 & 0.97 & 0.71 \\
\hline S3 & 0.0 & 2.5 & 0.00 & 0.01 & 0.00 & 0.01 & 0.04 & 0.03 & 0.23 & 0.06 & 0.82 & 0.12 & 1.00 & 0.17 \\
\hline S4 & 0.0 & 3.0 & 0.00 & 0.00 & 0.01 & 0.01 & 0.01 & 0.01 & 0.02 & 0.02 & 0.03 & 0.03 & 0.03 & 0.03 \\
\hline S5 & 0.0 & 6.0 & 0.13 & 0.00 & 0.39 & 0.00 & 0.87 & 0.01 & 0.95 & 0.07 & 0.96 & 0.34 & 0.99 & 0.77 \\
\hline S6 & 0.0 & 11.6 & 0.21 & 0.01 & 0.55 & 0.01 & 0.94 & 0.05 & 0.97 & 0.18 & 0.96 & 0.63 & 0.96 & 0.82 \\
\hline S7 & 0.0 & 126.0 & 0.16 & 0.02 & 0.37 & 0.04 & 0.80 & 0.12 & 0.86 & 0.29 & 0.79 & 0.58 & 0.76 & 0.67 \\
\hline A1 & 6.18 & 113.9 & 0.27 & 0.07 & 0.33 & 0.13 & 0.57 & 0.30 & 0.81 & 0.45 & 0.97 & 0.55 & 0.98 & 0.64 \\
\hline $\mathrm{A} 2$ & 2.0 & 9.0 & 0.19 & 0.04 & 0.50 & 0.03 & 0.91 & 0.09 & 0.97 & 0.25 & 0.99 & 0.73 & 1.00 & 0.93 \\
\hline A3 & 2.0 & 9.0 & 0.16 & 0.04 & 0.49 & 0.04 & 0.89 & 0.08 & 0.98 & 0.24 & 0.99 & 0.75 & 1.00 & 0.95 \\
\hline $\mathrm{A} 4$ & .5 & 2.2 & 0.06 & 0.01 & 0.12 & 0.01 & 0.52 & 0.04 & 0.89 & 0.13 & 1.00 & 0.24 & 1.00 & 0.40 \\
\hline A5 & 1.5 & 7.5 & 0.20 & 0.00 & 0.53 & 0.01 & 0.90 & 0.02 & 0.95 & 0.10 & 0.98 & 0.49 & 0.99 & 0.84 \\
\hline A6 & 2.0 & 21.2 & 0.28 & 0.01 & 0.55 & 0.02 & 0.92 & 0.07 & 0.98 & 0.22 & 0.97 & 0.60 & 0.96 & 0.74 \\
\hline A7 & 3.16 & 23.8 & 0.30 & 0.03 & 0.47 & 0.04 & 0.83 & 0.19 & 0.96 & 0.41 & 0.97 & 0.71 & 0.97 & 0.82 \\
\hline A8 & 3.8 & 40.7 & 0.27 & 0.05 & 0.45 & 0.07 & 0.81 & 0.22 & 0.94 & 0.42 & 0.98 & 0.66 & 0.98 & 0.76 \\
\hline
\end{tabular}


Table 3: Sample estimates of $\tau$ and $\kappa$

\begin{tabular}{|c|c|c|c|c|c|c|c|c|c|c|c|c|c|c|}
\hline & $\tau$ & 100 & 200 & 500 & 1000 & 2500 & 5000 & $\kappa$ & 100 & 200 & 500 & 1000 & 2500 & 5000 \\
\hline \multicolumn{15}{|l|}{$\rho=0$} \\
\hline S1 & 0.0 & 0.01 & -0.00 & -0.00 & 0.00 & 0.00 & -0.00 & 3.0 & 2.95 & 2.95 & 3.00 & 2.99 & 3.00 & 3.00 \\
\hline $\mathrm{S} 2$ & 0.0 & -0.01 & 0.02 & -0.02 & 0.01 & -0.02 & -0.02 & 9.0 & 5.38 & 6.11 & 6.66 & 7.88 & 7.87 & 8.12 \\
\hline $\mathrm{S} 3$ & 0.0 & 0.01 & 0.00 & 0.00 & 0.00 & 0.00 & 0.00 & 2.5 & 2.47 & 2.49 & 2.49 & 2.50 & 2.50 & 2.50 \\
\hline $\mathrm{S} 4$ & 0.0 & -0.01 & -0.00 & 0.00 & -0.00 & -0.00 & -0.00 & 3.0 & 2.96 & 2.97 & 2.99 & 2.99 & 3.00 & 3.00 \\
\hline $\mathrm{S} 5$ & 0.0 & -0.02 & -0.02 & 0.00 & 0.01 & -0.00 & 0.00 & 6.0 & 4.95 & 5.39 & 5.63 & 5.75 & 5.90 & 5.88 \\
\hline S6 & 0.0 & 0.03 & 0.02 & 0.02 & -0.00 & -0.01 & -0.01 & 11.6 & 6.70 & 7.66 & 8.92 & 9.80 & 10.71 & 11.09 \\
\hline $\mathrm{S} 7$ & 0.0 & 0.03 & -0.04 & 0.06 & -0.01 & -0.03 & -0.08 & 126.0 & 8.96 & 12.49 & 17.62 & 19.64 & 28.66 & 35.93 \\
\hline A1 & 6.18 & 3.15 & 3.81 & 4.44 & 4.82 & 5.34 & 5.71 & 113.9 & 17.24 & 25.71 & 37.63 & 46.55 & 63.72 & 79.20 \\
\hline $\mathrm{A} 2$ & 2.0 & 1.78 & 1.90 & 1.95 & 1.97 & 1.99 & 1.99 & 9.0 & 7.14 & 8.05 & 8.47 & 8.70 & 8.91 & 8.93 \\
\hline A3 & 2.0 & 1.76 & 1.89 & 1.98 & 1.97 & 2.00 & 1.99 & 9.0 & 6.98 & 7.94 & 8.85 & 8.70 & 9.02 & 8.91 \\
\hline A4 & .5 & 0.48 & 0.50 & 0.51 & 0.51 & 0.51 & 0.51 & 2.2 & 2.19 & 2.21 & 2.22 & 2.22 & 2.22 & 2.22 \\
\hline A5 & 1.5 & 1.33 & 1.43 & 1.47 & 1.49 & 1.51 & 1.52 & 7.5 & 5.86 & 6.53 & 6.90 & 7.16 & 7.30 & 7.42 \\
\hline A 6 & 2.0 & 1.34 & 1.49 & 1.72 & 1.80 & 1.87 & 1.97 & 21.2 & 8.30 & 10.00 & 12.66 & 14.14 & 15.74 & 18.68 \\
\hline A7 & 3.16 & 2.33 & 2.60 & 2.84 & 3.01 & 3.11 & 3.13 & 23.8 & 10.81 & 13.64 & 16.98 & 19.83 & 21.92 & 22.58 \\
\hline A8 & 3.8 & 2.58 & 2.89 & 3.21 & 3.56 & 3.66 & 3.72 & 40.7 & 12.65 & 16.43 & 20.86 & 27.93 & 31.22 & 32.50 \\
\hline \multicolumn{15}{|c|}{$\rho=.5$} \\
\hline S1 & 0.0 & 0.00 & -0.00 & -0.00 & 0.00 & 0.00 & 0.00 & 3.0 & 2.91 & 2.94 & 2.98 & 2.99 & 2.99 & 3.00 \\
\hline $\mathrm{S} 2$ & 0.0 & -0.02 & 0.02 & -0.02 & 0.01 & -0.01 & -0.01 & 9.0 & 4.31 & 4.81 & 5.16 & 5.91 & 5.88 & 6.09 \\
\hline S3 & 0.0 & 0.00 & 0.01 & 0.00 & 0.00 & -0.00 & -0.00 & 2.5 & 2.63 & 2.67 & 2.68 & 2.70 & 2.70 & 2.70 \\
\hline $\mathrm{S} 4$ & 0.0 & -0.01 & -0.01 & 0.00 & 0.00 & -0.00 & -0.00 & 3.0 & 2.91 & 2.94 & 2.98 & 2.99 & 3.00 & 3.00 \\
\hline S5 & 0.0 & -0.02 & -0.01 & -0.00 & 0.00 & -0.00 & -0.00 & 6.0 & 4.05 & 4.34 & 4.56 & 4.64 & 4.75 & 4.73 \\
\hline S6 & 0.0 & 0.03 & 0.01 & 0.01 & -0.00 & -0.01 & -0.01 & 11.6 & 5.06 & 5.72 & 6.51 & 7.08 & 7.61 & 7.87 \\
\hline $\mathrm{S} 7$ & 0.0 & 0.02 & -0.05 & 0.05 & -0.00 & -0.03 & -0.06 & 126.0 & 6.43 & 8.57 & 11.65 & 12.91 & 18.27 & 22.72 \\
\hline A1 & 6.18 & 2.23 & 2.77 & 3.27 & 3.57 & 3.95 & 4.24 & 113.9 & 10.92 & 16.11 & 23.49 & 29.03 & 39.22 & 48.85 \\
\hline $\mathrm{A} 2$ & 2.0 & 1.21 & 1.35 & 1.42 & 1.45 & 1.47 & 1.47 & 9.0 & 5.14 & 5.80 & 6.18 & 6.36 & 6.51 & 6.52 \\
\hline A3 & 2.0 & 1.21 & 1.35 & 1.45 & 1.45 & 1.48 & 1.47 & 9.0 & 5.09 & 5.76 & 6.39 & 6.38 & 6.57 & 6.52 \\
\hline A4 & .5 & 0.35 & 0.36 & 0.37 & 0.38 & 0.38 & 0.38 & 2.2 & 2.43 & 2.47 & 2.51 & 2.52 & 2.53 & 2.53 \\
\hline A5 & 1.5 & 0.96 & 1.05 & 1.09 & 1.10 & 1.12 & 1.13 & 7.5 & 4.53 & 5.04 & 5.32 & 5.45 & 5.55 & 5.65 \\
\hline A6 6 & 2.0 & 0.99 & 1.09 & 1.27 & 1.34 & 1.39 & 1.46 & 21.2 & 6.00 & 7.10 & 8.72 & 9.65 & 10.68 & 12.42 \\
\hline A7 & 3.16 & 1.65 & 1.90 & 2.10 & 2.22 & 2.31 & 2.32 & 23.8 & 7.31 & 9.17 & 11.29 & 13.01 & 14.33 & 14.68 \\
\hline A 8 & 3.8 & 1.85 & 2.09 & 2.36 & 2.62 & 2.71 & 2.75 & 40.7 & 8.44 & 10.67 & 13.54 & 17.74 & 19.83 & 20.60 \\
\hline \multicolumn{15}{|c|}{$\rho=.8$} \\
\hline S1 & 0.0 & -0.00 & 0.00 & -0.00 & -0.00 & 0.00 & 0.00 & 3.0 & 2.80 & 2.90 & 2.95 & 2.99 & 2.98 & 2.99 \\
\hline $\mathrm{S} 2$ & 0.0 & -0.01 & 0.01 & -0.01 & 0.01 & -0.01 & -0.01 & 9.0 & 3.21 & 3.53 & 3.72 & 4.02 & 4.03 & 4.12 \\
\hline S3 & 0.0 & 0.00 & 0.01 & 0.00 & 0.00 & 0.00 & 0.00 & 2.5 & 2.71 & 2.79 & 2.84 & 2.88 & 2.88 & 2.89 \\
\hline $\mathrm{S} 4$ & 0.0 & -0.01 & -0.02 & 0.00 & 0.00 & -0.00 & 0.00 & 3.0 & 2.78 & 2.89 & 2.95 & 2.98 & 2.99 & 3.00 \\
\hline $\mathrm{S} 5$ & 0.0 & -0.01 & 0.00 & -0.01 & 0.00 & -0.00 & 0.00 & 6.0 & 3.14 & 3.35 & 3.50 & 3.57 & 3.63 & 3.63 \\
\hline S6 & 0.0 & 0.02 & 0.00 & 0.00 & 0.00 & -0.00 & -0.01 & 11.6 & 3.48 & 3.81 & 4.18 & 4.44 & 4.64 & 4.78 \\
\hline $\mathrm{S} 7$ & 0.0 & -0.01 & -0.05 & 0.03 & 0.01 & -0.02 & -0.04 & 126.0 & 3.92 & 4.80 & 6.03 & 6.53 & 8.49 & 10.13 \\
\hline A1 & 6.18 & 0.84 & 1.36 & 1.81 & 2.06 & 2.31 & 2.51 & 113.9 & 5.01 & 6.87 & 9.86 & 12.09 & 15.84 & 19.67 \\
\hline $\mathrm{A} 2$ & 2.0 & 0.20 & 0.49 & 0.70 & 0.79 & 0.85 & 0.86 & 9.0 & 3.71 & 3.90 & 4.07 & 4.19 & 4.25 & 4.27 \\
\hline A3 & 2.0 & 0.20 & 0.48 & 0.73 & 0.79 & 0.85 & 0.86 & 9.0 & 3.72 & 3.86 & 4.15 & 4.19 & 4.26 & 4.26 \\
\hline A4 & .5 & 0.32 & 0.27 & 0.25 & 0.24 & 0.23 & 0.23 & 2.2 & 2.77 & 2.79 & 2.82 & 2.82 & 2.83 & 2.82 \\
\hline A5 & 1.5 & 0.42 & 0.52 & 0.61 & 0.64 & 0.66 & 0.67 & 7.5 & 3.17 & 3.46 & 3.73 & 3.82 & 3.90 & 3.97 \\
\hline A 6 & 2.0 & 0.56 & 0.62 & 0.76 & 0.78 & 0.83 & 0.87 & 21.2 & 3.67 & 4.23 & 4.95 & 5.29 & 5.77 & 6.45 \\
\hline A7 & 3.16 & 0.53 & 0.88 & 1.14 & 1.27 & 1.35 & 1.37 & 23.8 & 4.07 & 4.85 & 5.76 & 6.47 & 7.09 & 7.20 \\
\hline A8 & 3.8 & 0.67 & 0.98 & 1.29 & 1.50 & 1.59 & 1.63 & 40.7 & 4.40 & 5.19 & 6.46 & 8.08 & 9.03 & 9.37 \\
\hline
\end{tabular}


Table 4: Size and Power of the Test Normality

\begin{tabular}{|c|c|c|c|c|c|c|c|c|c|c|c|c|c|c|}
\hline \multicolumn{3}{|c|}{$\rho=.0$} & \multicolumn{2}{|c|}{$\mathrm{T}=100$} & \multicolumn{2}{|c|}{$\mathrm{T}=200$} & \multicolumn{2}{|c|}{$\mathrm{T}=500$} & \multicolumn{2}{|c|}{$\mathrm{T}=1000$} & \multicolumn{2}{|c|}{$\mathrm{T}=2500$} & \multicolumn{2}{|c|}{$\mathrm{T}=5000$} \\
\hline & $\tau$ & $\kappa$ & $\widehat{\pi}_{34}$ & $\widehat{\mu}_{34}$ & $\widehat{\pi}_{34}$ & $\widehat{\mu}_{34}$ & $\widehat{\pi}_{34}$ & $\widehat{\mu}_{34}$ & $\widehat{\pi}_{34}$ & $\widehat{\mu}_{34}$ & & $\widehat{\mu}_{34}$ & & $\widehat{\mu}_{34}$ \\
\hline S1 & 0.0 & 3.0 & 0.04 & 0.02 & 0.09 & 0.03 & 0.09 & 0.03 & 0.09 & 0.03 & 0.07 & 0.03 & 0.06 & 0.04 \\
\hline S2 & 0.0 & 9.0 & 0.04 & 0.02 & 0.11 & 0.08 & 0.39 & 0.30 & 0.61 & 0.56 & 0.81 & 0.76 & 0.89 & 0.80 \\
\hline S3 & 0.0 & 2.5 & 0.25 & 0.13 & 0.57 & 0.31 & 0.90 & 0.72 & 0.98 & 0.95 & 1.00 & 1.00 & 1.00 & 1.00 \\
\hline $\mathrm{S} 4$ & 0.0 & 3.0 & 0.04 & 0.02 & 0.08 & 0.02 & 0.07 & 0.03 & 0.07 & 0.03 & 0.06 & 0.03 & 0.07 & 0.02 \\
\hline S5 & 0.0 & 6.0 & 0.06 & 0.02 & 0.13 & 0.10 & 0.53 & 0.42 & 0.82 & 0.74 & 0.94 & 0.89 & 0.98 & 0.96 \\
\hline S6 & 0.0 & 11.6 & 0.07 & 0.04 & 0.20 & 0.13 & 0.49 & 0.43 & 0.66 & 0.60 & 0.85 & 0.77 & 0.91 & 0.83 \\
\hline S7 & 0.0 & 126.0 & 0.08 & 0.04 & 0.19 & 0.14 & 0.37 & 0.34 & 0.55 & 0.46 & 0.63 & 0.56 & 0.71 & 0.61 \\
\hline A1 & 6.18 & 113.9 & 0.06 & 0.46 & 0.71 & 0.55 & 0.98 & 0.69 & 1.00 & 0.80 & 1.00 & 0.84 & 1.00 & 0.90 \\
\hline A2 & 2.0 & 9.0 & 0.31 & 0.69 & 1.00 & 0.88 & 1.00 & 0.98 & 1.00 & 1.00 & 1.00 & 1.00 & 1.00 & 1.00 \\
\hline A3 & 2.0 & 9.0 & 0.33 & 0.71 & 1.00 & 0.88 & 1.00 & 0.97 & 1.00 & 1.00 & 1.00 & 1.00 & 1.00 & 1.00 \\
\hline A4 & .5 & 2.2 & 0.74 & 0.98 & 1.00 & 1.00 & 1.00 & 1.00 & 1.00 & 1.00 & 1.00 & 1.00 & 1.00 & 1.00 \\
\hline A5 & 1.5 & 7.5 & 0.71 & 0.59 & 0.99 & 0.82 & 1.00 & 0.97 & 1.00 & 0.99 & 1.00 & 1.00 & 1.00 & 1.00 \\
\hline A6 & 2.0 & 21.2 & 0.19 & 0.25 & 0.50 & 0.52 & 0.86 & 0.76 & 0.97 & 0.86 & 1.00 & 0.94 & 1.00 & 0.94 \\
\hline $\mathrm{A} 7$ & 3.16 & 23.8 & 0.18 & 0.59 & 0.95 & 0.74 & 1.00 & 0.86 & 1.00 & 0.92 & 1.00 & 0.97 & 1.00 & 0.98 \\
\hline$\overline{\mathrm{A} 8}$ & 3.8 & 40.7 & 0.13 & 0.55 & 0.92 & 0.68 & 1.00 & 0.82 & 1.00 & 0.88 & 1.00 & 0.94 & 1.00 & 0.98 \\
\hline \multicolumn{3}{|c|}{$\rho \rho=.5$} & & & & & & & & & & & & \\
\hline S1 & 0.0 & 3.0 & 0.03 & 0.01 & 0.05 & 0.01 & 0.08 & 0.03 & 0.08 & 0.03 & 0.08 & 0.03 & 0.05 & 0.03 \\
\hline S2 & 0.0 & 9.0 & 0.02 & 0.02 & 0.05 & 0.05 & 0.20 & 0.16 & 0.48 & 0.42 & 0.76 & 0.72 & 0.87 & 0.79 \\
\hline S3 & 0.0 & 2.5 & 0.06 & 0.02 & 0.20 & 0.06 & 0.48 & 0.23 & 0.68 & 0.40 & 0.93 & 0.79 & 1.00 & 0.98 \\
\hline S4 & 0.0 & 3.0 & 0.03 & 0.01 & 0.06 & 0.02 & 0.08 & 0.03 & 0.09 & 0.03 & 0.07 & 0.03 & 0.05 & 0.02 \\
\hline S5 & 0.0 & 6.0 & 0.03 & 0.02 & 0.05 & 0.05 & 0.27 & 0.19 & 0.61 & 0.55 & 0.91 & 0.84 & 0.98 & 0.96 \\
\hline S6 & 0.0 & 11.6 & 0.05 & 0.04 & 0.09 & 0.07 & 0.34 & 0.28 & 0.60 & 0.56 & 0.82 & 0.74 & 0.89 & 0.82 \\
\hline S7 & 0.0 & 126.0 & 0.07 & 0.06 & 0.13 & 0.10 & 0.34 & 0.31 & 0.50 & 0.43 & 0.62 & 0.55 & 0.70 & 0.61 \\
\hline$\overline{\mathrm{A} 1}$ & 6.18 & 113.9 & 0.47 & 0.51 & 0.94 & 0.57 & 0.99 & 0.68 & 1.00 & 0.79 & 1.00 & 0.84 & 1.00 & 0.90 \\
\hline$\overline{\mathrm{A} 2}$ & 2.0 & 9.0 & 0.53 & 0.54 & 1.00 & 0.83 & 1.00 & 0.96 & 1.00 & 1.00 & 1.00 & 1.00 & 1.00 & 1.00 \\
\hline A3 & 2.0 & 9.0 & 0.58 & 0.56 & 1.00 & 0.82 & 1.00 & 0.95 & 1.00 & 0.99 & 1.00 & 1.00 & 1.00 & 1.00 \\
\hline A4 & .5 & 2.2 & 0.52 & 0.34 & 0.95 & 0.85 & 1.00 & 1.00 & 1.00 & 1.00 & 1.00 & 1.00 & 1.00 & 1.00 \\
\hline A5 & 1.5 & 7.5 & 0.33 & 0.35 & 0.89 & 0.71 & 1.00 & 0.94 & 1.00 & 0.98 & 1.00 & 1.00 & 1.00 & 1.00 \\
\hline $\mathrm{A} 6$ & 2.0 & 21.2 & 0.13 & 0.19 & 0.38 & 0.43 & 0.78 & 0.73 & 0.95 & 0.84 & 1.00 & 0.93 & 1.00 & 0.94 \\
\hline $\mathrm{A} 7$ & 3.16 & 23.8 & 0.55 & 0.54 & 0.98 & 0.70 & 1.00 & 0.85 & 1.00 & 0.91 & 1.00 & 0.97 & 1.00 & 0.98 \\
\hline A8 & 3.8 & 40.7 & 0.53 & 0.53 & 0.99 & 0.67 & 1.00 & 0.81 & 1.00 & 0.87 & 1.00 & 0.94 & 1.00 & 0.97 \\
\hline \multicolumn{3}{|c|}{$\rho \bar{\rho} \rho=.8$} & & & & & & & & & & & & \\
\hline S1 & 0.0 & 3.0 & 0.00 & 0.00 & 0.01 & 0.01 & 0.02 & 0.01 & 0.05 & 0.03 & 0.08 & 0.04 & 0.06 & 0.03 \\
\hline S2 & 0.0 & 9.0 & 0.01 & 0.01 & 0.01 & 0.01 & 0.02 & 0.02 & 0.07 & 0.05 & 0.24 & 0.22 & 0.60 & 0.55 \\
\hline S3 & 0.0 & 2.5 & 0.00 & 0.00 & 0.02 & 0.00 & 0.07 & 0.02 & 0.12 & 0.05 & 0.22 & 0.09 & 0.29 & 0.14 \\
\hline $\mathrm{S} 4$ & 0.0 & 3.0 & 0.01 & 0.01 & 0.01 & 0.00 & 0.04 & 0.01 & 0.07 & 0.03 & 0.09 & 0.03 & 0.07 & 0.03 \\
\hline S5 & 0.0 & 6.0 & 0.00 & 0.00 & 0.01 & 0.01 & 0.02 & 0.03 & 0.05 & 0.04 & 0.25 & 0.18 & 0.67 & 0.60 \\
\hline S6 & 0.0 & 11.6 & 0.01 & 0.01 & 0.01 & 0.02 & 0.04 & 0.05 & 0.11 & 0.12 & 0.50 & 0.47 & 0.76 & 0.71 \\
\hline S7 & 0.0 & 126.0 & 0.02 & 0.02 & 0.02 & 0.03 & 0.06 & 0.08 & 0.18 & 0.19 & 0.49 & 0.44 & 0.61 & 0.56 \\
\hline A1 & 6.18 & 113.9 & 0.07 & 0.11 & 0.19 & 0.35 & 0.92 & 0.62 & 0.99 & 0.74 & 1.00 & 0.83 & 1.00 & 0.89 \\
\hline $\mathrm{A} 2$ & 2.0 & $\begin{array}{l}9.0 \\
\end{array}$ & 0.10 & 0.04 & 0.08 & 0.11 & 0.57 & 0.63 & 0.99 & 0.94 & 1.00 & 1.00 & 1.00 & 1.00 \\
\hline A3 & 2.0 & 9.0 & 0.11 & 0.04 & 0.07 & 0.11 & 0.59 & 0.64 & 1.00 & 0.92 & 1.00 & 1.00 & 1.00 & 1.00 \\
\hline $\mathrm{A} 4$ & .5 & 2.2 & 0.04 & 0.04 & 0.05 & 0.04 & 0.26 & 0.18 & 0.67 & 0.46 & 0.97 & 0.91 & 1.00 & 1.00 \\
\hline A5 & 1.5 & 7.5 & 0.01 & 0.02 & 0.04 & 0.09 & 0.51 & 0.49 & 0.94 & 0.85 & 1.00 & 0.99 & 1.00 & 1.00 \\
\hline A6 & 2.0 & 21.2 & 0.02 & 0.03 & 0.04 & 0.09 & 0.30 & 0.37 & 0.70 & 0.67 & 0.97 & 0.89 & 1.00 & 0.94 \\
\hline A7 & 3.16 & 23.8 & 0.04 & 0.05 & 0.08 & 0.21 & 0.84 & 0.70 & 1.00 & 0.85 & 1.00 & 0.95 & 1.00 & 0.98 \\
\hline A8 & 3.8 & 40.7 & 0.04 & 0.07 & 0.12 & 0.26 & 0.90 & 0.70 & 0.99 & 0.82 & 1.00 & 0.93 & 1.00 & 0.96 \\
\hline
\end{tabular}


Table 5: Macroeconomic Data

\begin{tabular}{|c|c|c|c|c|c|c|}
\hline Sample & Series & $\widehat{\pi}_{3}$ & $\widehat{\pi}_{4}$ & $\widehat{\pi}_{34}$ & $\widehat{\tau}$ & $\widehat{\kappa}$ \\
\hline $71: 1-97: 12$ & Canada-U.S. Ex. Rate & 1.559 & 0.371 & 2.685 & 0.226 & 3.139 \\
$71: 1-97: 12$ & German-U.S. Ex. Rate & -0.646 & 1.128 & 1.799 & -0.134 & 3.499 \\
$71: 1-97-12$ & Japan-U.S. Ex. Rate & -2.379 & 2.103 & 8.522 & -0.481 & 3.905 \\
$48: 1-97: 12$ & Unemployment Rate & 0.926 & 1.235 & 7.913 & 0.308 & 8.621 \\
$46: 1-97: 12$ & Ind. Prod. & 1.223 & 1.675 & 5.074 & 0.994 & 13.274 \\
$59: 1-97: 4$ & Inflation (GDP) & 1.771 & 0.253 & 3.076 & 0.870 & 3.284 \\
$59: 1-97: 4$ & GDP & -1.408 & 1.410 & 2.305 & -0.561 & 4.717 \\
$47: 1-97: 12$ & Inflation (CPI) & 3.186 & 1.666 & 10.104 & 0.942 & 4.491 \\
81:10:30-96:05:10: & 30 day Int. Rate & -0.564 & 2.704 & 8.496 & -0.415 & 11.861 \\
$80: 11: 03-98: 01: 19$ & M2 & -0.100 & 0.310 & 0.199 & -0.017 & 3.116 \\
$59: 3-96: 4$ & Con. Durables & -1.868 & 1.697 & 3.545 & -0.791 & 5.023 \\
$59: 3-96: 4$ & Con. Non-Durables & 0.569 & 1.443 & 4.181 & 0.212 & 4.721 \\
$46: 1-96: 11$ & Employment & -1.560 & 1.589 & 3.747 & -0.280 & 3.733 \\
$49: 3-97: 4$ & Investment & -1.525 & 1.291 & 2.568 & -0.732 & 5.254 \\
$46: 1-97: 12$ & Manu. Employment & -1.845 & 1.710 & 2.624 & -1.644 & 10.543 \\
$46: 1-97: 12$ & Non Manu. Employment & 0.395 & 1.260 & 4.468 & 0.117 & 5.857 \\
$59 ; 3-97: 4$ & Final Sales & -0.034 & 1.506 & 2.794 & -0.017 & 5.404 \\
$59: 3-97: 4$ & Non-Resid. Invest & -1.549 & 1.072 & 2.805 & -0.409 & 3.680 \\
$59: 3-97: 4$ & Resid. Invest & -0.955 & 1.341 & 2.249 & -0.457 & 5.134 \\
$90: 01: 02-96: 12: 31$ & Stock Returns (V) & -2.764 & 3.248 & 10.859 & -0.481 & 5.187 \\
$90: 01: 02-96: 12: 31$ & Stock Returns (E) & -3.931 & 3.455 & 16.166 & -0.990 & 6.943 \\
\hline
\end{tabular}

The $5 \%$ critical value for $\pi_{3}$ and $\pi_{4}$ is 1.96 , and for $\pi_{34}$ is 5.99 . 